\title{
Ferromagnetic resonance spectra of permalloy nano-ellipses as building blocks for complex magnonic lattices
}

\author{
Wonbae Bang, ${ }^{1,2^{*}}$ F. Montoncello, ${ }^{3}$ M. T. Kaffash, ${ }^{4}$ A. Hoffmann, ${ }^{2 \dagger}$ J. B. Ketterson ${ }^{1,5}$, and M. \\ B. Jungfleisch, ${ }^{4}$ \\ ${ }^{1}$ Department of Physics and Astronomy, Northwestern University, Evanston, IL 60208, USA \\ ${ }^{2}$ Materials Science Division, Argonne National Laboratory, Argonne, IL 60439, USA \\ ${ }^{3}$ Dipartimento di Fisica e Scienze della Terra, Università di Ferrara, I-44122 Ferrara, Italy \\ ${ }^{4}$ Department of Physics and Astronomy, University of Delaware, Newark, DE 19716, USA \\ ${ }^{5}$ Department of Electrical and Computer Engineering, Northwestern University, Evanston, IL \\ 60208, USA
}

\begin{abstract}
We report the experimental and theoretical characterization of the angulardependent magnetic field and microwave frequency dependence of the response of isolated mesoscopic permalloy nano-ellipses for geometries in which the static magnetic field is both parallel to and perpendicular to the microwave magnetic field. These spectra form a basis for interpreting the ferromagnetic resonance (FMR) spectra of lattices based on such structures, including those containing frustrated regions, such as artificial spin ice structures.
\end{abstract}

\section{INTRODUCTION}

Mesoscopic segments having an elongated (e.g., elliptical or bar-like) cross section have been used to assemble interconnected lattices such as those used in artificial spin ice studies, which is a topic of considerable recent activity. ${ }^{1-4}$ The magnetic response of the individual segments themselves is also of interest, and the case of permalloy ellipses has been studied via Brillouin scattering for magnetic fields along the in-plane principal axes, ${ }^{5-8}$ and via the timeresolved magneto-optic Kerr effect (TR-MOKE). ${ }^{9}$ We recently completed a study of the microwave response of three-fold, $120^{\circ}$-symmetric, clusters of such ellipses ${ }^{10}$ and their angular dependence $^{11}$; our future intension is to incorporate these vertices into honeycomb or Kagome spin-ice lattices. But prior to this, it is important to characterize the behavior of the isolated elements used to assemble these structures, so as to examine the extent to which the responses of

\footnotetext{
* Present address: Institute of Advanced Materials, LG Chem, Daejeon 34122, Korea

$\dagger$ Present address: Department of Materials Science and Engineering, University of Illinois at Urbana-Champaign, Urbana, IL 61801 USA

* Corresponding author:mbj@udel.edu
} 
the individual ellipses govern the overall response of the clusters and, ultimately, lattices assembled from them. In so doing, we can advance ferromagnetic resonance (FMR) as a tool that complements magnetic force microscopy and static magnetization studies, where the latter only probes the net magnetization of a structure.

Here we report the angular dependence of in-plane FMR measurements using a vector network analyzer (VNA) on 15-nm thick permalloy nano-ellipses with lateral dimensions of 500 $\times 200 \mathrm{~nm}^{2}$ in the range of 2 to $10 \mathrm{GHz}$. Our discussion consists of three parts: i) the observed angular dependence of the spectra of single nano-ellipse arrays, ii) a comparison of this data with micromagnetic calculations using the dynamical matrix method where excellent agreement with the experiment is found, and iii) a comparison of the single ellipse data with that of three- and four-fold nano-ellipse clusters, showing that, semi-quantitatively, the latter can be seen as a superposition of the single ellipse data.

\section{EXPERIMENTAL}

Most of our devices consist of a square array of well separated (lattice constant $=1880$ $\mathrm{nm})$ permalloy nano-ellipses patterned over the central strip of a coplanar waveguide (CPW). A limited number of samples were also fabricated with the ellipses between signal and ground lines. It has earlier been shown that having metallic contact with the underlying guide results in maximal coupling, thereby achieving high overall sensitivity. ${ }^{12}$ In the CPWs, the central line has $20 \mu \mathrm{m}$ width, and the two ground lines have $40 \mu \mathrm{m}$. The spacing between the central line and each ground line is $8 \mu \mathrm{m}$. The devices are fabricated by the following process: First, the pattern for the CPWs was defined using a laser writer followed by electron beam evaporation of $5 \mathrm{~nm}$ of $\mathrm{Ti}$ and $120 \mathrm{~nm}$ of $\mathrm{Au}$ on an underlying intrinsic $\mathrm{Si}$ substrate having a $300 \mathrm{~nm} \mathrm{\textrm {SiO } _ { 2 }}$ layer; the latter insures electrical isolation of the resulting guide elements. In the next step, a 15-nm thick permalloy ( $\mathrm{Py}, \mathrm{Ni}_{80} \mathrm{Fe}_{20}$ ) nano-ellipse array was fabricated by electron beam lithography and electron beam evaporation, using a lift-off process. The thicknesses of the deposited materials were monitored by a quartz crystal microbalance during the evaporation: the rates were $\sim 0.2$ $\AA / \mathrm{sec}$ for $\mathrm{Ti}, \sim 1.4 \AA / \mathrm{sec}$ for $\mathrm{Au}$, and $\sim 0.4 \AA / \mathrm{sec}$ for Py, respectively. The pressure in the chamber during the metal depositions was $\sim 3 \times 10^{-7}$ Torr. 
The probe station in our instrumental set up cannot be rotated. Our goals in the present work included: i) the angular dependence with respect to the angle between the ellipse axis and static magnetic field direction, and ii) studies of the polarization dependence, in which the microwave field is perpendicular to, or parallel to the static magnetic field direction. To address these two behaviors, we fabricated two different types of CPWs: one set for which the static field was applied parallel to the guide axis and a second set in which it was perpendicular.

Figure 1 shows a schematic representation of the two geometries used for the polarization measurements. Fig. 1(a) shows configuration 1 where the static field is parallel to the guide axis. For the case shown here the Py ellipses were patterned with their long axes perpendicular to the guide axis. The angle between the magnetic field and the ellipse axis is designated as $\theta_{\mathrm{H}}$; here $\theta_{\mathrm{H}}=0^{\circ}$.

Fig. 1(b) shows configuration 2 where the static field is perpendicular to the guide axis. For the case shown here the Py ellipses were patterned with their long axes parallel to the guide axis. The angle between the magnetic field and the ellipse axis is also designated as $\theta_{\mathrm{H}}$; here $\theta_{\mathrm{H}}=$ $90^{\circ}$. Although we designate the orientation of the ellipses as $\theta_{\mathrm{H}}$ for the two configurations, they correspond to the same field direction; however, the direction of the dynamic field differs by $90^{\circ}$ between the configurations.

To observe the angular dependence of the Py ellipses, multiple devices were prepared oriented at $\theta_{\mathrm{H}}=0^{\circ}, 15^{\circ}, 30^{\circ}, 45^{\circ}, 60^{\circ}, 75^{\circ}$ and $90^{\circ}$ relative to the guide axis. Scanning electron microscopy (SEM) images of devices in the first configuration are shown in Fig. 2 (a) to (g). Here the microwave field is perpendicular to the static field [red arrow in Fig. 1 (a) and Fig. 2]. To explore the polarization dependence, seven additional samples were prepared in configuration 2 where the CPW axis was perpendicular to the magnetic field, as shown in Fig. 1 (b). For these experiments, the ellipses were also oriented at $0^{\circ}, 15^{\circ}, 30^{\circ}, 45^{\circ}, 60^{\circ}, 75^{\circ}$, and $90^{\circ}$, with the magnetic field along the y-axis [blue arrow in Fig. 1(b) and Fig. 2], parallel to the r.f. driving field.

To probe the dynamic magnetic response of the ellipses, we performed broadband FMR measurements of the transmission parameter $S_{21}$ at a nominal microwave power of $0 \mathrm{dBm}$ using a vector network analyzer (VNA). ${ }^{13}$ The microwave absorption spectra were recorded using the 
following steps: First, the magnetic field was set at +3000 Oe, and the frequency swept between 2 and $10 \mathrm{GHz}$ to establish a baseline which was subtracted from the data gathered at all other fields. This field is sufficient to erase any prior history and establish a well-defined starting point for measuring the subsequent responses. In the next step, frequency sweeps were then carried out between 2 and $10 \mathrm{GHz}$ for discrete magnetic fields ranging between +900 and -900 Oe in steps of $10 \mathrm{Oe}$.

\section{DYNAMICAL MATRIX METHOD}

The theoretical calculations were performed using the dynamical matrix method (DMM). ${ }^{14,15}$ The DMM is based on the solution of the Hamilton equations for the precessional motion through the diagonalization of a dynamical matrix, which contains the second derivatives of the energy density with respect to the angular coordinates; all the contributions to the energy of the system are computed as interactions among the magnetic moments of the elemental (discretization) cells: the dipolar interaction (through the computation of the specific demagnetizing tensor corresponding to the geometry of the sample), the exchange interaction (among nearest neighbor elemental cells), and the Zeeman interaction (between the applied field and the magnetic moment of each elemental cell).

Advantages of the method are that a single calculation provides all the independent modes allowed by the mesh through which the sample is discretized (i.e., equal to the number of micromagnetic cells), independently of their symmetry and strength. Conversely, full simulations obtain results that depend on characteristics of the excitation (e.g. the polarization and profile) and hence require separate runs for each case, together with extended simulation times to detect weakly-coupled modes with the same precision. ${ }^{16,17}$ Moreover, if periodic boundary conditions are included, the calculation time are the same as for a single particle; conversely, in full micromagnetic simulations, it is necessary to include several primitive cells to accurately simulate the full periodic system, with a dramatic increase of the computation times. The computation times strongly depend on the system size (i.e., the number of active elemental cells), as well as the computer used. In our case, with an 8-CPU Intel ${ }^{\circledR}$ Xeon ${ }^{\circledR}$ E5462, the calculations typically run for approximately $300 \mathrm{~s}$ (3150 elemental cells). 
The following parameters of permalloy were used: saturation magnetization $\mathrm{M}_{\mathrm{s}}=650$ $\mathrm{kA} / \mathrm{m}$, exchange stiffness parameter $\mathrm{A}=1.00 \times 10^{-11} \mathrm{~J} / \mathrm{m}$, and gyromagnetic ratio, $\gamma=185 \mathrm{rad}$ $\mathrm{GHz} / \mathrm{T}$, and anisotropy coefficient $\mathrm{K}=0$ (no anisotropy).

\section{RESULTS AND DISCUSSION}

Figure 3 shows false color images of the FMR spectra for the case where the d.c. magnetic field is aligned along the $\mathrm{x}$-axis which is parallel to the CPW axis. The red solid lines show the results of the theoretical calculations and will be described in detail below. We again note that $\theta_{\mathrm{H}}$ is the angle between applied static magnetic field and the major axis of the ellipses, as shown in Fig. 3(a). At $\theta_{\mathrm{H}}=0^{\circ}$, the magnetization initially remains parallel to the field as it is reduced and the FMR frequency falls. When the field passes through zero, ${ }^{18}$ a metastable regime is entered in which the field and magnetization are oppositely aligned. At approximately -240 Oe in Fig. 3(b), an instability is encountered, where the magnetization abruptly switches by $180^{\circ}$, so as to again align parallel to the external field, but now in the $-\mathrm{x}$ direction. When this realignment occurs the FMR frequency abruptly increase to about $7 \mathrm{GHz}$; note this value is essentially identical to the value it had at +240 Oe.

The behavior at $\theta_{\mathrm{H}}=15^{\circ}, 30^{\circ}$, and $45^{\circ}$ shown in Fig. 3(c), (d), and (e) is quite similar to that at $0^{\circ}$, however the region of metastability is reduced. At $\theta_{\mathrm{H}}=60^{\circ}$ in Fig. 3(f), there is a field range where no FMR signal is observed ranging from about 0 to -225 Oe. This occurs because the static magnetization in this region has rotated such that it largely lies parallel to the $H_{f}$ of the $\mathrm{CPW}$, in which case the torque in the Landau-Lifshitz equation is greatly diminished.

Fig. 3(h) shows the data $\theta_{\mathrm{H}}=90^{\circ}$ which displays some additional features. At high fields, the magnetization is aligned with the short axis; this is the hard direction and corresponds to what is termed a leaf state. ${ }^{10,19}$ We find that the $90^{\circ}$ saturation field occurs at 640 Oe: at this field value, the magnetization parallel to the applied field is larger than $95 \%$ with respect to the saturation value, while the perpendicular one is lower than $10 \%$.

As the field is reduced from +900 Oe, the FMR frequency falls, but the magnetization initially stays aligned with the field. This holds down to a point where this alignment is no longer stable, at which point the magnetization tips in plane, to one side or the other; this occurs at approximately +450 Oe where a cusp occurs, after which the FMR frequency rises. As the field 
is reduced to zero, the magnetization rotates so as to lie parallel to the dynamic $H_{f}$, where the torque arising from the $H_{f}$ vanishes; the signal then vanishes accordingly. A black arrow at -380 Oe indicates a discontinuous behavior which approximately coincides with a feature in the calculations, to be discussed next [see Fig. 3(h)], that is assigned to a soft mode. ${ }^{8,20}$

The red solid lines in Fig. 3 show the results of the theoretical calculations for the nanoellipses with different angles corresponding to the experimental data, when the applied d.c. magnetic field lies along the CPW axis, i.e. the $\mathrm{x}$-axis. The calculations were performed using the DMM as described above, and the single nano-ellipse was discretized with $5 \times 5 \times 15 \mathrm{~nm}^{3}$ square-based elemental cells. Overall, the experimental data shown are in good agreement with the theoretical calculation.

Fig. 4 shows the data for our second configuration in which the CPWs have been rotated by $90^{\circ}$ and where the d.c. magnetic field is aligned along the $y$-axis which is perpendicular to the guide axis. We note again that $\theta_{\mathrm{H}}$ is the angle between the applied static magnetic field and the major axis of the nano-ellipse, as shown in Fig. 4(a). However, the intensities differ since the static and dynamic fields are now aligned. As an example, the signal in the missing field range of Fig. 3(f) is now particularly intense in Fig. 4(f).

In Fig. 4(h) the static magnetic field is along the minor axis of the ellipses. However, the magnetization favors the long axis at low fields. The dynamic field, which is here parallel to the static field, is then perpendicular to the magnetization yielding maximal torque, and hence the signal is large. The field also lies along the minor axis in Fig. 3(h) and again the magnetization is perpendicular to the static field. However here the dynamic field is parallel to the magnetization and the torque vanishes, consistent with the disappearance of the signal at low static fields. In Fig. 4(b) the field lies along the major axis of the ellipses; however, the dynamic field is again in the same direction leading to a vanishing torque. In Fig. 3(b) the magnetization and field are again parallel (or antiparallel) to the static field but the dynamic field is perpendicular and we have a large signal.

The DMM yields the full angular range of the modes. Experimentally, however, we see that, depending on the orientation between the dynamic in-plane field and the magnetization, one can highlight different regions of the curves. This suggests that if the dynamic field were to be perpendicular to the plane (i.e. out-of-plane), then the measurement would reproduce the full 
angular dependence. To test this hypothesis, we fabricated a sample with the ellipses patterned in the gap between the signal and the ground lines [see Fig. 5(a)]. Here the field is largely perpendicular to the plane of the ellipses, although due to the limited space their total number is smaller; this, together with the lack of direct metallic contact with the wave guide (known empirically to enhance coupling, see above), results in over-all weaker signals.

Figure 5 shows the data for the samples prepared (the patterning of the $90^{\circ}$ sample failed); here the applied magnetic field is parallel to the axis of the CPW (the y-axis). Note that the signal amplitude for the $60^{\circ}$ data shown in Fig. 5(f) is relatively uniform as the field is swept and the magnetization rotates in the sample plane. This is to be contrasted with the $60^{\circ}$ data shown in Fig. 3(f), where the field is also parallel to the guide axes, but now there is no "missing region" in the vicinity of -150 Oe. A similar contrast is seen at $75^{\circ}$ although the overall amplitude is much weaker with this sample.

The motivation for the present study is to identify whether individual building blocks, such as ellipses or bars, could be useful for the understanding of the overall dynamic response of networks assembled from them. As examples, Fig. 6(e) and (f) show SEM images of $120^{\circ}$ symmetric clusters formed from three nano-ellipses having the same dimensions as those studied here and where the distance of closest approach is $\sim 60 \mathrm{~nm}$. The orientation in Fig. 6(f) is rotated by $90^{\circ}$ counterclockwise from Fig. 6(e).

The FMR spectra for the $120^{\circ}$ (three-fold) cluster with the magnetic field parallel to the long axis of an ellipse is shown in Fig. 6(a). On the other hand, figure 6(c) shows the sum of the spectra of the single ellipses oriented at $0^{\circ}$ [taken from Fig. 3(b)] and $60^{\circ}$ [from Fig. 3(f)]. Note that the spectrum shown in Fig. 6(a) closely corresponds to a superposition of Fig. 3(b) and 3(f) with the branch involving two symmetry equivalent ellipses having an enhanced intensity.

Fig. 6(b) shows the spectrum for the $120^{\circ}$ cluster with the field perpendicular to the long axis of an ellipse. On the other hand, Fig. 6(d) shows a sum of the spectra shown in Fig. 3(h) and 3(d). Again, we see that the spectrum of the cluster closely corresponds to a superposition of that for the individual nano-ellipses with the intensities higher for the branches involving two symmetry-equivalent ellipses.

Figures 7(f) and (g) show a $90^{\circ}$ symmetric cluster formed from four nano-ellipses with the same dimensions for the magnetic field at $0^{\circ}$ and $45^{\circ}$, respectively. Figure 7(a) shows the spectrum of the $90^{\circ}$ (four-fold) symmetric nano-ellipse clusters with the field parallel to a 
principal axis, while Fig. 7(c) shows the sum of the spectra shown in Fig. 3(b) and (h). Again, the superposition "principle" approximately holds. In Fig. 7(a), three branches are observed. Branch 1 corresponds to Fig. 3(b); however, the branch is shifted about $1 \mathrm{GHz}$ to lower frequency. Branch 2 corresponds to Fig. 3(h); however, in low negative fields (indicating by a black arrow), the mode increases with decreasing magnetic field. Branch 3 in the lowest frequency regime is not observed in the sum of spectra of the single nano-ellipses in Fig. 7(c). Finally, Fig. 7(b) shows the spectrum of the four-fold clusters with the field rotated by $45^{\circ}$, while Fig. 7(d) shows the $45^{\circ}$ spectrum taken from Fig. 3(e); note the close correspondence and that, by symmetry, there is now only a single branch, although the spectrum is shifted to lower frequency by about $1 \mathrm{GHz}$.

\section{CONCLUSIONS}

We have performed a broad-band study of the angular dependence of the ferromagnetic resonance (FMR) spectra of permalloy nano-ellipses having dimensions of $500 \times 200 \times 15 \mathrm{~nm}^{3}$. By orienting the wave guide axis parallel and perpendicular to the d.c. magnetic field, the dependence of the coupling on the microwave polarization is exhibited. It is demonstrated that a detailed understanding of the behavior of these individual ellipses qualitatively explains the response of clusters of ellipses, although with some quantitative shifts occur due to the interaction between ellipses within the cluster. We propose that this superposition principle can be applied to more complex structures involving lattices formed from such clusters, such as artificial spin-ice structures. 


\section{ACKNOWLEDGEMENTS}

Work at Northwestern, including experimental design and FMR measurements was supported under NSF grant DMR 1507058. Work at Delaware, data analysis and manuscript preparation, was supported under NSF Grant No. 1833000. Device fabrication was carried out at Argonne and supported by the U.S. Department of Energy, Office of Science, Materials Science and Engineering Division. Lithography was carried out at the Center for Nanoscale Materials, an Office of Science user facility, which is supported by DOE, Office of Science, Basic Energy Science under Contract No. DE-AC02-06CH11357. 


\section{REFERENCES}

${ }^{1}$ I. Gilbert, C. Nisoli, and P. Schiffer, Phys. Today 69(7), 54 (2016).

${ }^{2}$ M. Krawczyk and D. Grundler, J. Phys.-Condens. Mat. 26, 123202 (2014).

${ }^{3}$ C. Nisoli, R. Moessner, and P. Schiffer, Rev. Mod. Phys. 85, 1473 (2013).

${ }^{4}$ R. F. Wang, C. Nisoli, R. S. Freitas, J. Li, W. McConville, B. J. Cooley, M. S. Lund, N. Samarth, C. Leighton, V. H. Crespi, and P. Schiffer, Nature 439(7074), 303 (2006).

${ }^{5}$ M. Grimsditch, F. Y. Fradin, Y. Ji, A. Hoffmann, R. E. Camley, V. Metlushko, and V. Novosad, Phys. Rev. Lett. 96, 047401 (2006).

${ }^{6}$ G. Gubbiotti, G. Carlotti, T. Okuno, M. Grimsditch, L. Giovannini, F. Montoncello, and F. Nizzoli, Phys. Rev. B 72, 184419 (2005).

${ }^{7}$ Y. Li, G. Gubbiotti, F. Casoli, F. J. T. Goncalves, S. A. Morley, M. C. Rosamond, E. H. Linfield, C. H. Marrows, S. McVitie, and R. L. Stamps, J. Phys. D Appl. Phys. 50(1), 015003 (2017).

${ }^{8}$ F. Montoncello, L. Giovannini, F. Nizzoli, P. Vavassori, M. Grimsditch, T. Ono, G. Gubbiotti, S. Tacchi, and G. Carlotti, Phys. Rev. B 76, 024426 (2007).

${ }^{9}$ Y. Yahagi, C. R. Berk, B. D. Harteneck, S. D. Cabrini, and H. Schmidt, Appl. Phys. Lett. 104, 162406 (2014).

${ }^{10}$ F. Montoncello, L. Giovannini, W. Bang, J. B. Ketterson, M. B. Jungfleisch, A. Hoffmann, B. W. Farmer, and L. E. De Long, Phys. Rev. B 97, 014421 (2018).

${ }^{11}$ W. Bang, F. Montoncello, M. B. Jungfleisch, A. Hoffmann, L. Giovannini, and J. B. Ketterson, Phys. Rev. B 99, 014415 (2019).

${ }^{12}$ M. B. Jungfleisch, W. Zhang, E. Iacocca, J. Sklenar, J. Ding, W. Jiang, S. Zhang, J. E. Pearson, V. Novosad, J. B. Ketterson, O. Heinonen, and A. Hoffmann, Phys. Rev. B 93, 100401(R) (2016).

${ }^{13}$ S. S. Kalarickal, P. Krivosik, M. Z. Wu, C. E. Patton, M. L. Schneider, P. Kabos, T. J. Silva, and J. P. Nibarger, J. Appl. Phys. 99, 093909 (2006).

${ }^{14}$ L. Giovannini, F. Montoncello, and F. Nizzoli, Phys. Rev. B 75, 024416 (2007).

${ }^{15}$ M. Grimsditch, L. Giovannini, F. Montoncello, F. Nizzoli, G. K. Leaf, and H. G. Kaper, Phys. Rev. B 70, 054409 (2004).

${ }^{16}$ O. Dmytriiev, V. V. Kruglyak, M. Franchin, H. Fangohr, L. Giovannini, and F. Montoncello, Phys. Rev. B 87, 174422 (2013).

${ }^{17}$ F. Montoncello, L. Giovannini, F. Nizzoli, R. Zivieri, G. Consolo, and G. Gubbiotti, J. Magn. Magn. Mater. 322(16), 2330 (2010).

${ }^{18}$ We recall that for the simple case of a uniformly magnetized ellipsoid having three different demagnetization coefficients, the FMR frequency can be non zero even in the absence of an external field.

${ }^{19}$ J. K. Ha, R. Hertel, and J. Kirschner, Phys. Rev. B 67, 064418 (2003).

${ }^{20}$ D. Kuźma, F. Montoncello, P. Sobieszczyk, A. Wal, A. Giovannini, and P. Zieliński, J. Appl. Phys. 124, 223902 (2018). 


\section{CAPTIONS}

Fig. 1. Illustrations of the permalloy nano-ellipse devices, drawn with their long axes parallel to the guide axis, for the two different measurement geometries for which: (a) the magnetic field is applied along the $\mathrm{x}$-axis ( $\mathrm{H} / / \mathrm{CPW}$ axis) (b) the magnetic field is applied along the $\mathrm{y}$-axis $(\mathrm{H} \perp$ CPW axis).

Fig. 2. (a) to (g) SEM images of all the seven permalloy nano-ellipse array devices that were prepared; $\theta_{\mathrm{H}}$ describes the angle between the applied field (solid line), which is either parallel to or perpendicular to the CPW axis [red $\left(\mathrm{H}_{\text {para }}\right)$ and blue $\left(\mathrm{H}_{\text {perp }}\right)$ arrow] and a major axis of the nano-ellipses for angles (dotted line), (a) $0^{\circ}$, (b) $15^{\circ}$, (c) $30^{\circ}$, (d) $45^{\circ}$, (e) $60^{\circ}$, (f) $75^{\circ}$, and (g) $90^{\circ}$. Note that the angle $\theta_{\mathrm{h}}$ is the angle of the driving microwave magnetic field $\mathrm{H}_{\mathrm{f}}$ with respect to the major axis of the nano-ellipses. The permalloy nano-ellipses are written on the central strip of a CPW. The ellipses have dimensions of: $500 \times 200 \mathrm{~nm}^{2}$ and a thickness of $15 \mathrm{~nm}$. The square lattice has a spacing $(a)$ of $1880 \mathrm{~nm}$.

Fig. 3. (a) Schematic of a device (here drawn with the ellipses parallel to guide axis); also shown is the angle $\left(\theta_{\mathrm{H}}\right)$ between the magnetic field and major axis of the nano-ellipses. (b) to (h) show the FMR spectra obtained for permalloy nano-ellipses prepared with different angles $\left(\theta_{\mathrm{H}}=0^{\circ}\right.$, $15^{\circ}, 30^{\circ}, 45^{\circ}, 60^{\circ}, 75^{\circ}$ and $90^{\circ}$ ). The magnetic field was applied along the $\mathrm{x}$-axis (parallel to the CPW axis). Here the frequency is swept between 2 and $10 \mathrm{GHz}$ at successive magnetic fields ranging between +900 and -900 Oe in 10 Oe steps. White arrows indicate the direction in which the applied field was swept during the measurements. The black arrows indicate features due to soft modes that precede the subsequent transition. Theoretical calculations of the frequency as a function of the magnetic field for nano-ellipses with different angles $\left(\theta_{\mathrm{H}}=0^{\circ}, 15^{\circ}, 30^{\circ}, 45^{\circ}, 60^{\circ}\right.$, $75^{\circ}$ and $90^{\circ}$ ) when the d.c. magnetic field is parallel to the CPW axis. The sweeping direction was from +900 Oe -900 Oe. Please note that for the theoretical results a non-uniform field spacing was used. Depending on how fast the frequency slope varied, the spacing was chosen between 200 Oe to 10 Oe.

Fig. 4. (a) Schematic of a device showing the angle $\left(\theta_{\mathrm{H}}\right)$ between the magnetic field and the major axis of the nano-ellipses (here drawn with the ellipse axis perpendicular to the guide axis). (b) to (h) show FMR spectra obtained for permalloy nano-ellipses prepared with different angles $\left(\theta_{\mathrm{H}}=0^{\circ}, 15^{\circ}, 30^{\circ}, 45^{\circ}, 60^{\circ}, 75^{\circ}\right.$ and $\left.90^{\circ}\right)$, but with the applied magnetic field perpendicular to the axis of the CPW (the y-axis). The frequency is swept between 2 and $10 \mathrm{GHz}$ at fixed magnetic fields ranging between +900 and -900 Oe in 10 Oe steps. White arrows indicate the direction in which the applied field was swept during the measurements.

Fig. 5. (a) Schematic of the permalloy nano-ellipses patterned within the gap between the ground and the signal lines of the CPW. Figs. (b) to (e) show FMR spectra obtained for permalloy nanoellipses prepared with different angles $\left(0^{\circ}, 45^{\circ}, 60^{\circ}\right.$, and $\left.75^{\circ}\right)$ with the applied magnetic field 
parallel to the axis of the CPW (the y-axis). The frequency is swept between 2 and $10 \mathrm{GHz}$ at fixed magnetic fields ranging between +900 and -900 Oe in 10 Oe steps. White arrows indicate the direction in which the applied field was swept during the measurements.

Fig. 6. FMR spectra obtained for $120^{\circ}$ (three-fold) nano-ellipse clusters; in (a) the applied static magnetic field is parallel to the long axis of an ellipse and in (b) the magnetic field is perpendicular to the long axis of an ellipse. The spectra shown in Fig. 6(c) and (d) are the sum of the spectra of the single nano-ellipses shown in Fig. 3. Figs. 6(e) and 6(f) show SEM images of the nano-ellipse clusters; the clusters in 6(f) are rotated $90^{\circ}$ counterclockwise from those in 6(e).

Fig. 7. FMR spectra obtained for $90^{\circ}$ (four-fold) nano-ellipse clusters; in (a) the applied static magnetic field is parallel to the long axis of two ellipses, and in (b) the magnetic field is rotated $45^{\circ}$ from the long axis of an ellipse. Figs. 7(c) is taken from Fig.'s 3 (b) and (h), and 7(d) is taken from Fig. 3(e). SEM images of the nano-ellipse clusters; (e) is rotated $45^{\circ}$ counterclockwise from (f). 


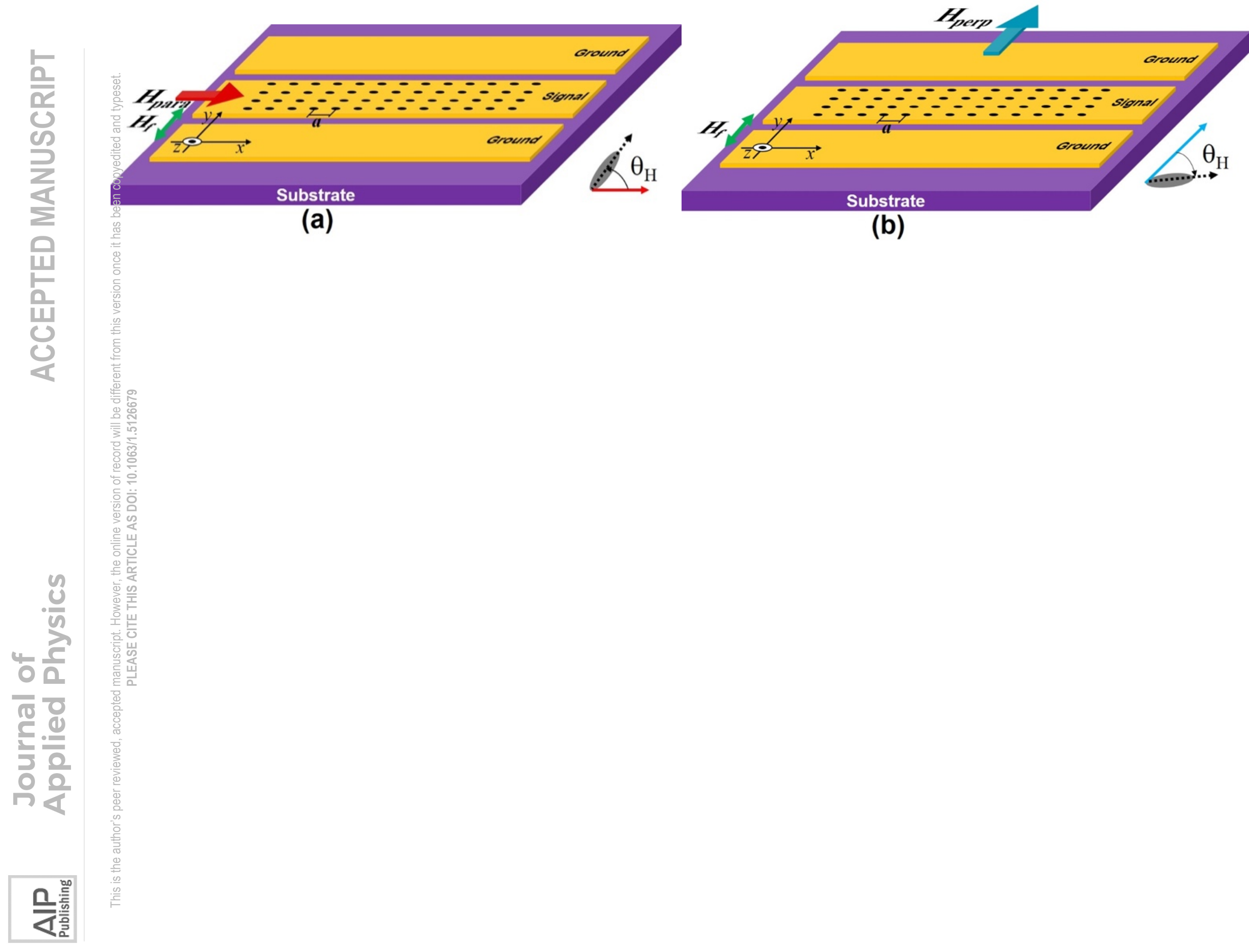


(a) $0^{\circ}$

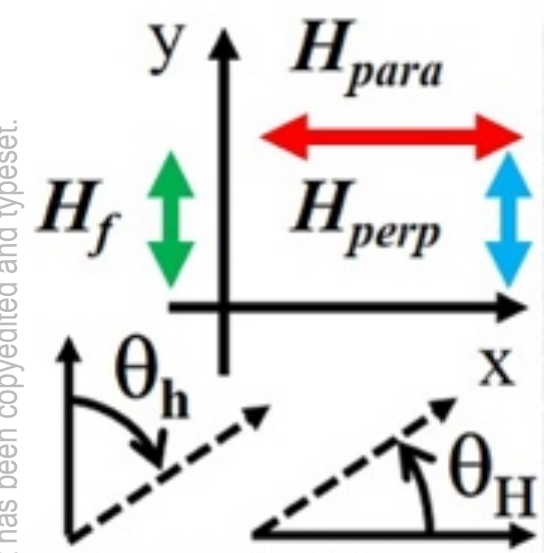

$500 \mathrm{~nm}$
(d) $45^{\circ}$
(e) $60^{\circ}$

(b) $15^{\circ}$

(c) $30^{\circ}$

\section{$a=1880 \mathrm{~nm}$}

(f) $75^{\circ}$

(g) $90^{\circ}$ 

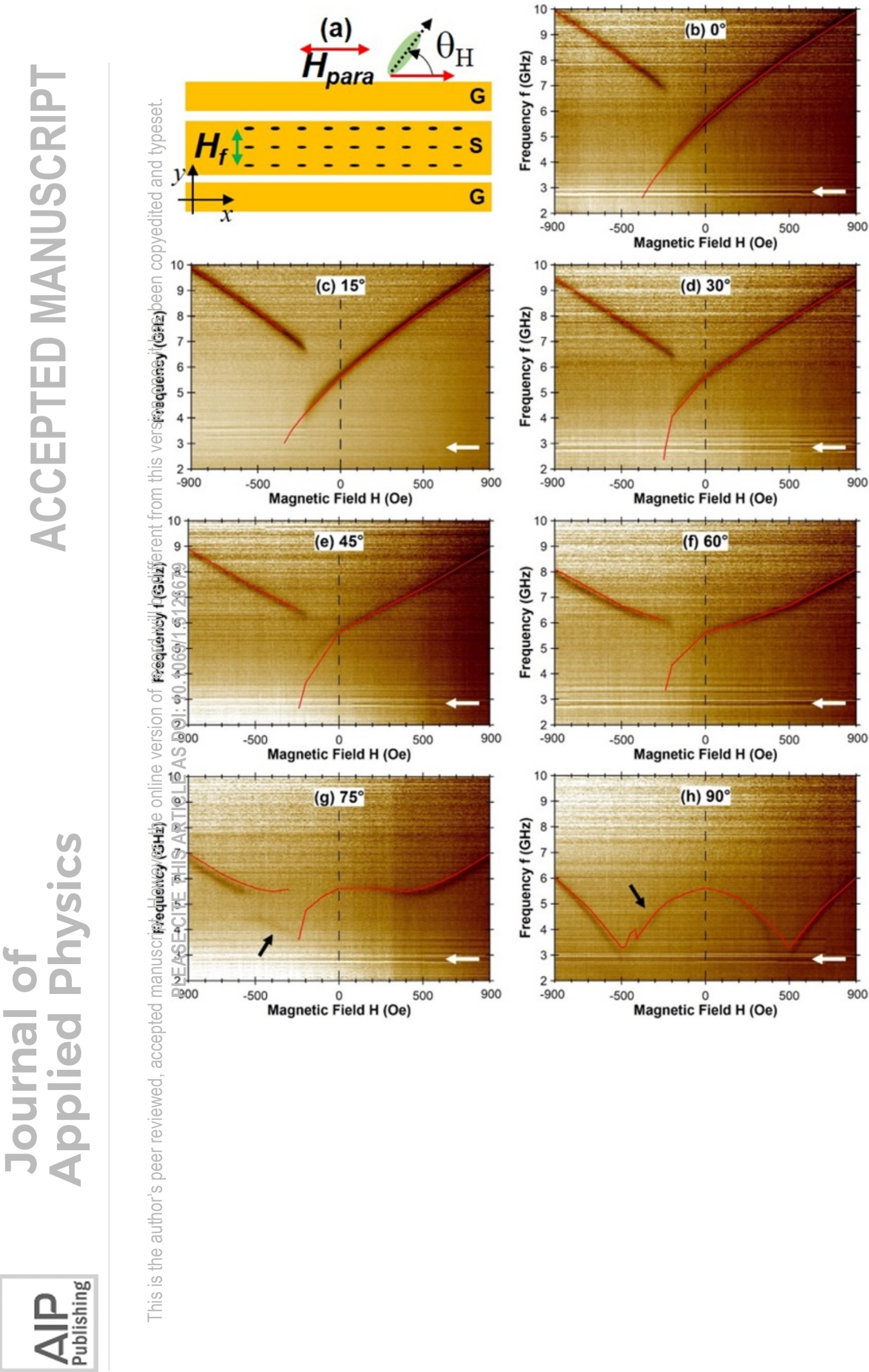

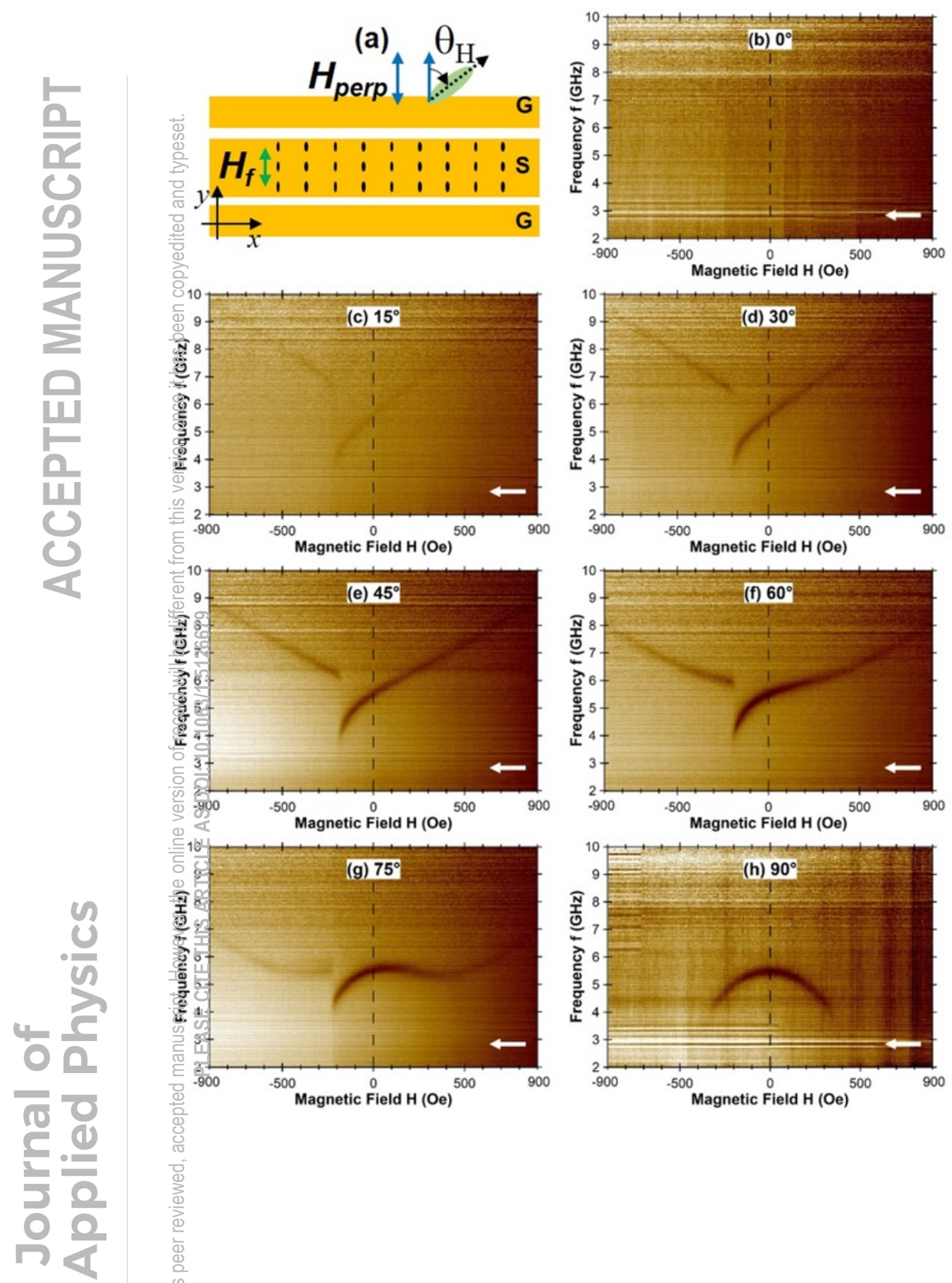
(a)

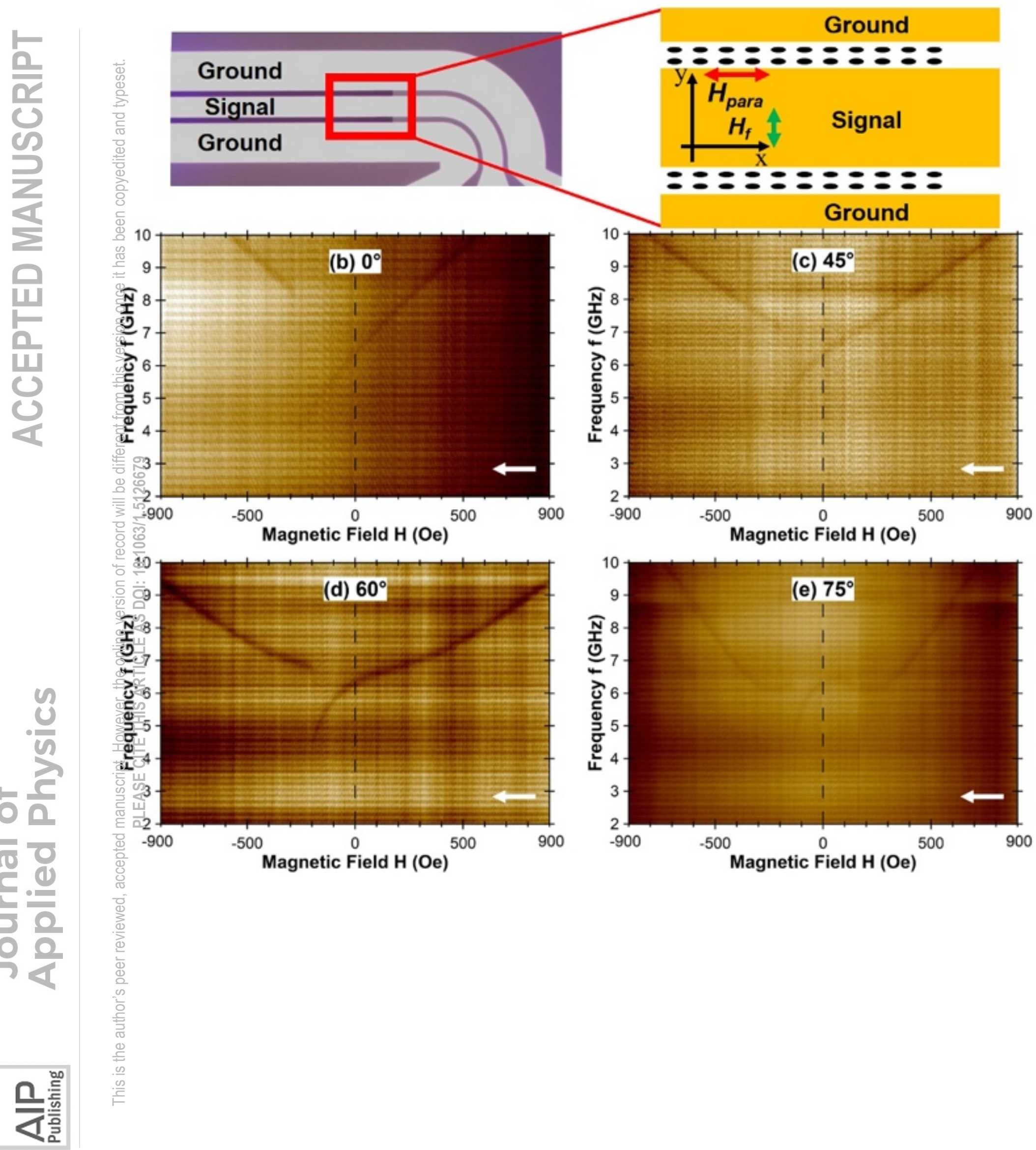



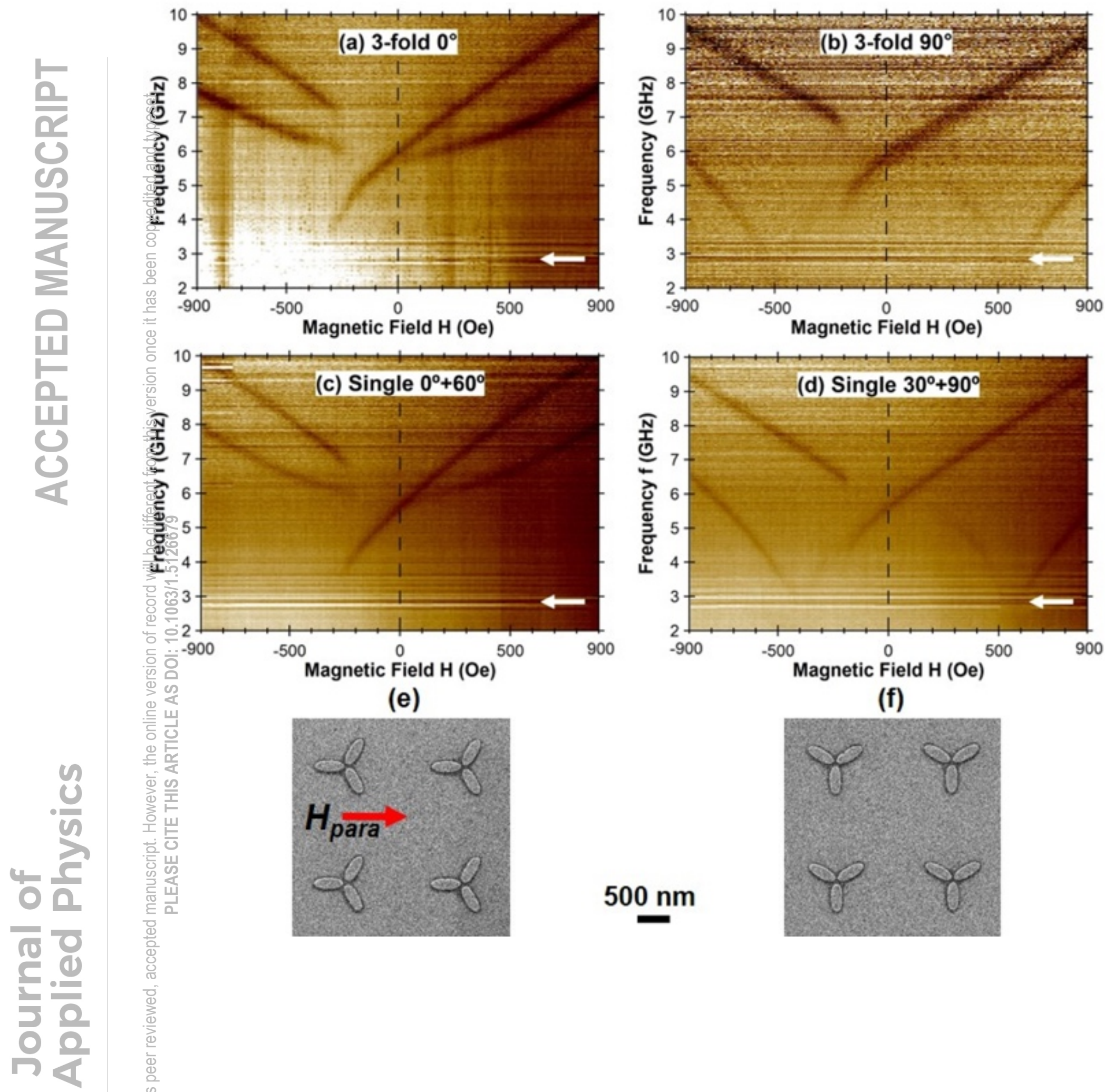

(e)

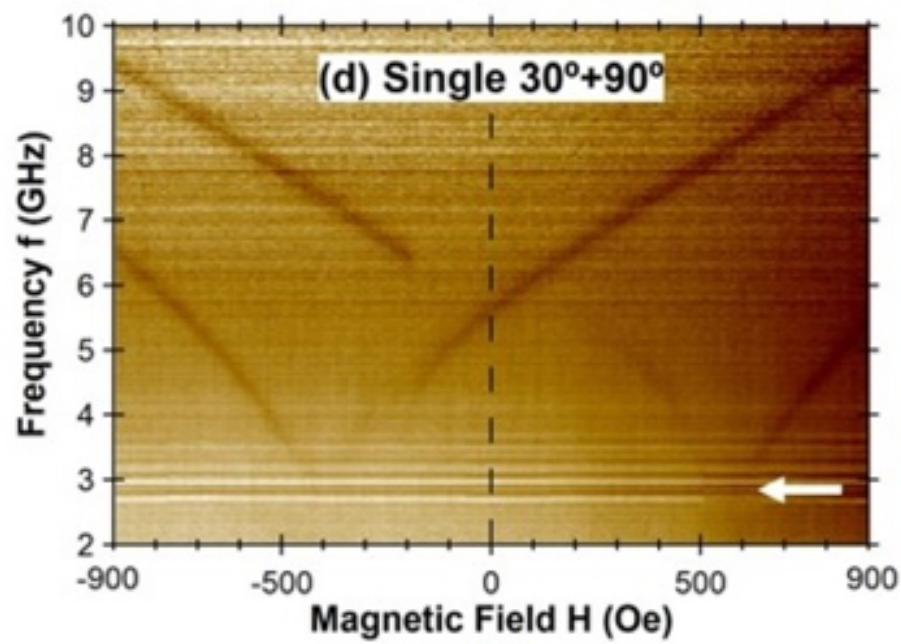

(f)

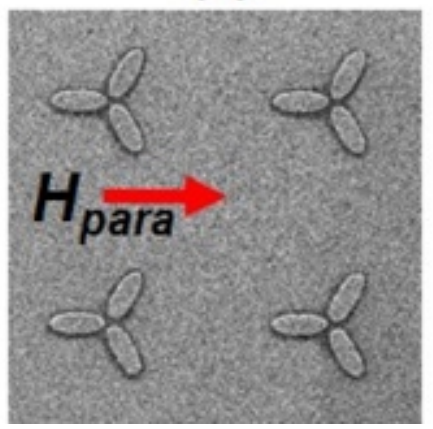

$500 \mathrm{~nm}$

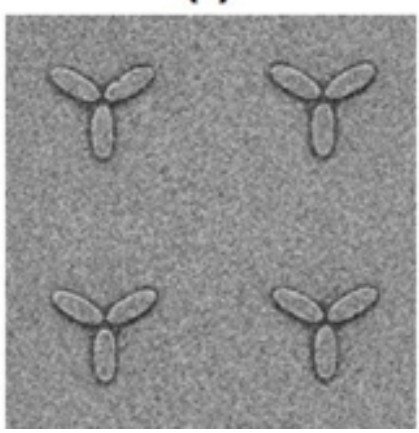



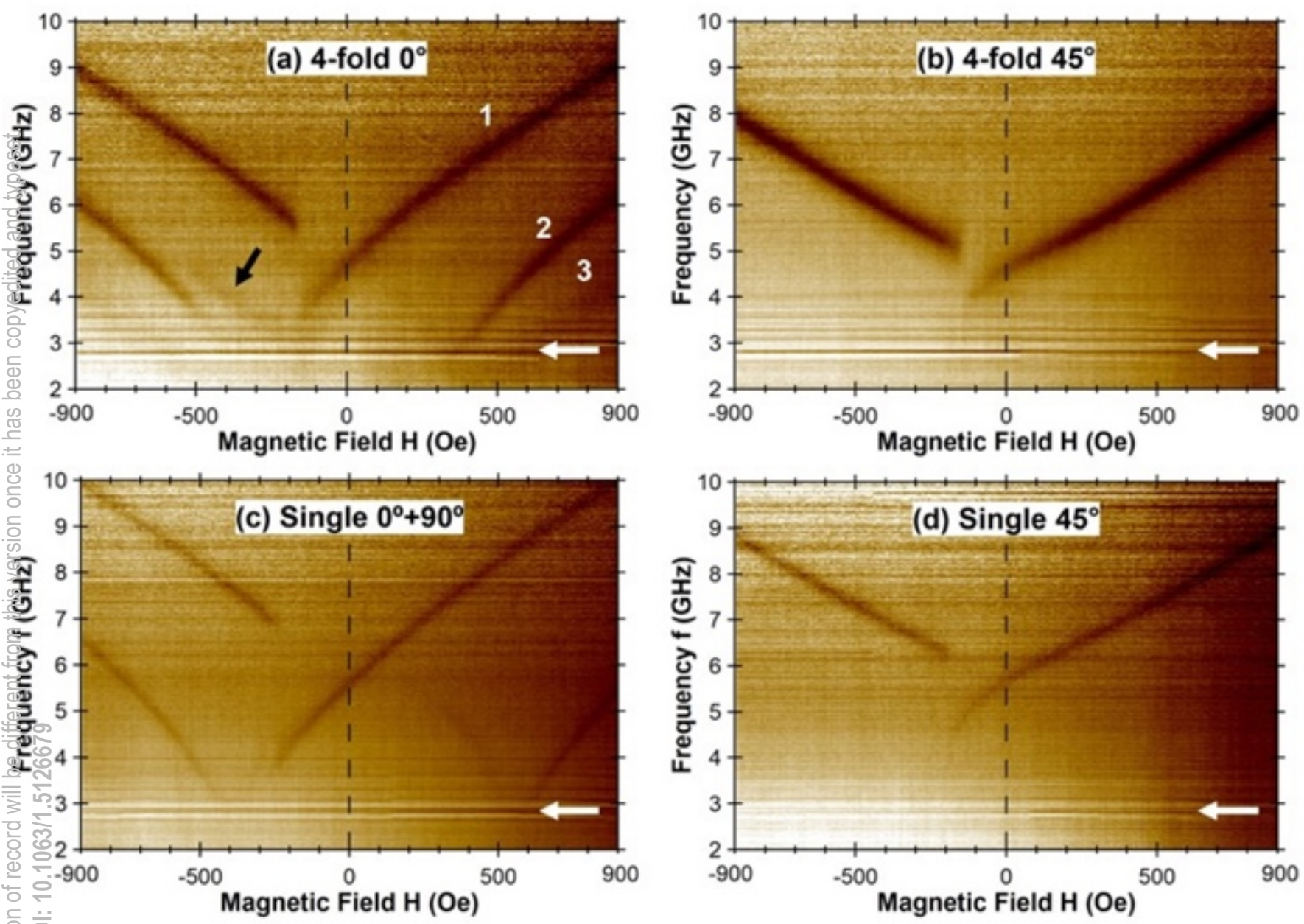

(e)

(f)
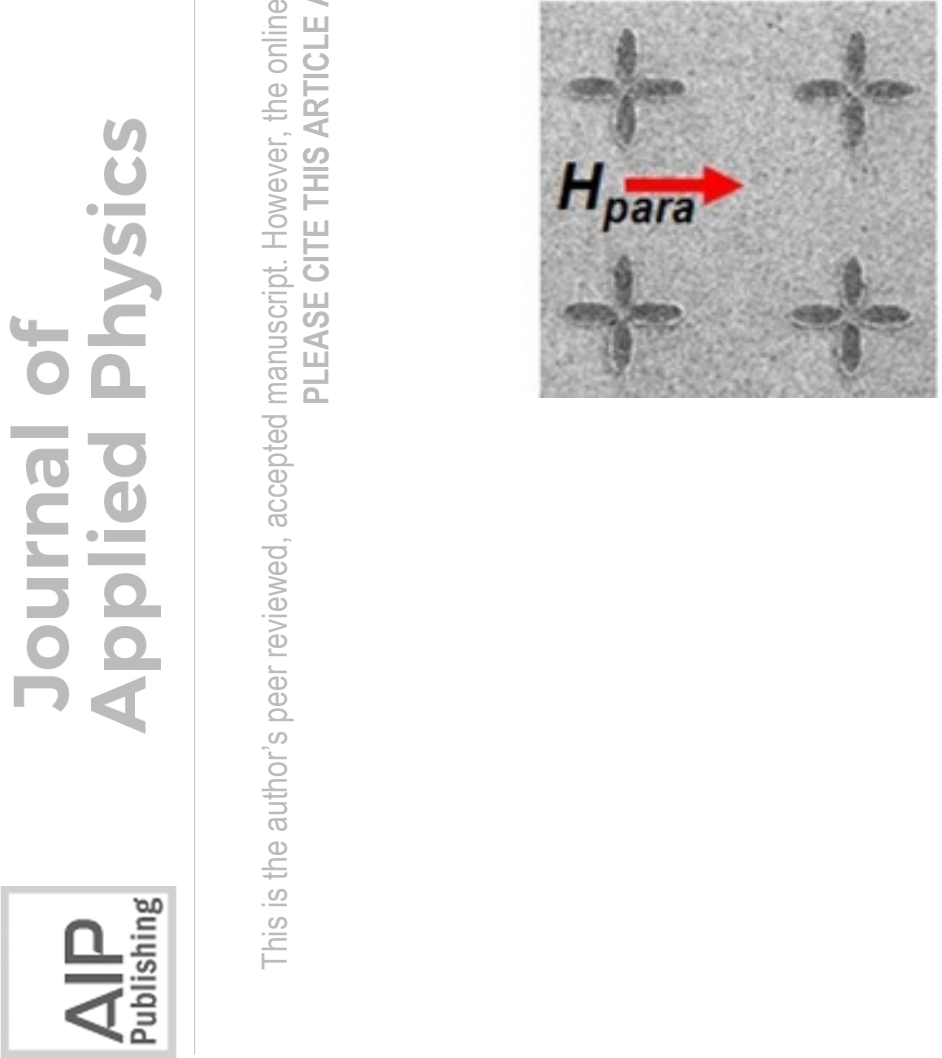